\title{
BMJ Open Quality Promoting appropriate utilisation of laboratory tests for inflammation at an academic medical centre
}

\author{
Kristen J Bartlett, ${ }^{1}$ Ann P Vo, ${ }^{2}$ Justin Rueckert, ${ }^{3}$ Christina Wojewoda, ${ }^{3}$ \\ Elizabeth H Steckel, ${ }^{4}$ Justin Stinnett-Donnelly, ${ }^{5}$ Allen B Repp (D) ${ }^{5}$
}

To cite: Bartlett KJ, Vo AP, Rueckert J, et al. Promoting appropriate utilisation of laboratory tests for inflammation at an academic medical centre. BMJ Open Quality 2020;9:e000788. doi:10.1136/ bmjoq-2019-000788

Received 5 August 2019 Revised 28 January 2020 Accepted 11 February 2020

\section{Check for updates}

C Author(s) (or their employer(s)) 2020. Re-use permitted under CC BY-NC. No commercial re-use. See rights and permissions. Published by BMJ.

${ }^{1}$ Department of Medicine, Maine Medical Center, Portland, Maine, USA

${ }^{2}$ Adult Primary Care, US Department of Veterans Affairs, Burlington, Vermont, USA ${ }^{3}$ Department of Pathology and Laboratory Medicine, University of Vermont Medical Center, Burlington, Vermont, USA ${ }^{4}$ Jeffords Institute for Quality, University of Vermont Medical Center, Burlington, Vermont, USA

${ }^{5}$ Department of Medicine, University of Vermont Medical Center, Burlington, Vermont, USA

Correspondence to

Dr Allen B Repp;

allen.repp@uvmhealth.org

\section{ABSTRACT}

Erythrocyte sedimentation rate (ESR) and $\mathrm{C}$ reactive protein (CRP) are commonly ordered in clinical practice to evaluate for inflammation. CRP is a more sensitive and specific test for detecting acute phase inflammation, and the American Society for Clinical Pathology recommends ordering CRP rather than ESR to detect acute phase inflammation in patients with undiagnosed conditions. We sought to understand CRP and ESR ordering practices and reduce unnecessary use of ESR testing at our academic medical centre. We surveyed physician leaders in clinical areas with high utilisation of ESR testing to understand the drivers of potential overutilisation of these tests. Based on survey responses, we designed an intervention focused on education, clinical decision support within the electronic medical record and quarterly audit and feedback. We evaluated appropriateness of ESR ordering before and after the intervention via structured chart audit. Comparison of monthly rates of ESR tests during the preintervention and postintervention periods was conducted using interrupted time series analysis. Clinical habit and ease of test ordering were identified as key drivers of ESR overuse. Compared with the preintervention period, we observed a $33 \%$ reduction in the number of ESR tests per month and a $25 \%$ reduction in combined CRP and ESR tests per month during the postintervention period. This reduction corresponded to an annual avoidance of 2633 ESR tests with a corresponding estimated direct cost avoidance of \$23 701 annually. Although the rate of ESR testing decreased, there was no significant improvement in the clinical appropriateness of residual ESR test ordering following the intervention. A multifaceted intervention was associated with significant decreases in unnecessary ESR tests and concurrent ESR and CRP tests at our academic medical centre. Despite these reductions, there are continued opportunities to reduce inappropriate ESR testing.

\section{PROBLEM}

Erythrocyte sedimentation rate (ESR or sed rate) and $\mathrm{C}$ reactive protein (CRP) are commonly used tests in clinical practice to evaluate for inflammation. CRP has been shown to be a more sensitive and specific test when assessing acute phase inflammation. ${ }^{12}$ Recommendations from the American Society for Clinical Pathology, as part of the Choosing Wisely campaign, state 'Don't order an erythrocyte sedimentation rate (ESR) to look for inflammation in patients with undiagnosed conditions. Order a C-reactive protein (CRP) to detect acute phase inflammation'?

The initiative was conducted at a 562-bed, level 1 trauma, academic medical centre in New England. We observed high rates of ESR and combined ESR/CRP testing at our medical centre, leading to concerns about potential overuse of ESR. We speculated that combined, simultaneous ESR and CRP orders were rarely indicated and could provide a marker of unnecessary tests, since CRP is preferred for detecting acute inflammation in undiagnosed conditions, whereas ESR may be preferred for monitoring disease activity in some specific inflammatory conditions. We surmised that lack of knowledge regarding the test characteristics of ESR and CRP, ordering habits and the design of order entry within the electronic medical record (EMR) contributed to overuse of ESR testing. We hypothesised that interventions targeting specific drivers identified at our institution would decrease unnecessary ESR testing, improve clinical appropriateness of ESR testing and effect institutional cost savings.

We sought to understand the drivers for ESR use, reduce unnecessary ESR testing, assess changes in clinician ordering practices and estimate the impact on the costs of care delivery. Specifically, we aimed to reduce the total number of ESR tests per month and the number of combined ESR and CRP tests per month by $50 \%$ within 12 months of initiating the project. We also aimed to improve the clinical appropriateness of ESR ordering so that testing was used for specific inflammatory conditions rather than for acute phase inflammation in undiagnosed conditions.

\section{BACKGROUND}

CRP is a specific acute phase reactant associated with inflammation and infection, and ESR is a non-specific measure of the acute 
phase reaction. ESR is influenced by many factors, both inflammatory and non-inflammatory, such as blood viscosity, age, sex and other chronic conditions such as renal disease, obesity and heart failure. ${ }^{3}$ Thus, an elevated ESR has limited specificity for inflammation. ESR may remain elevated even after the CRP has returned to baseline. These characteristics make CRP the preferred test for detecting acute phase inflammation in patients with undiagnosed conditions. ${ }^{2}$ Simultaneous ordering of ESR and CRP has been shown to increase costs without evidence of improving diagnostic accuracy or patient outcomes. ${ }^{4}$ Interventions incorporating education, audit and feedback and computerised clinical decision support (CDS) have been associated with reductions in ESR testing in hospitals. ${ }^{5}$ The clinical appropriateness of residual ESR testing following such interventions and the estimated changes in costs of laboratory testing have not been reported.

\section{MEASUREMENT}

Our primary measures were the total number of ESR tests and the number of concurrent ESR and CRP tests performed each month by the laboratory at our academic medical centre. Concurrent testing was determined to be present if the ESR and CRP tests were performed on the same sample based on the accession number. The total number of complete blood count (CBC) tests performed each month was used as a control, as a CBC is a common test that reflects overall laboratory test volumes and is frequently used to evaluate for inflammation and infection. Thus, $\mathrm{CBC}$ testing rates provided a control for changes in patient volume and other temporal trends in test ordering practices. These data were collected on a monthly basis throughout our project.

We used an interrupted time series analysis with ordinary least squares regression to adjust for autocorrelation to compare the number of ESR, CRP and CBC tests per month in the preintervention period (1 September 2016-31 October 2017) and postintervention periods (1 November 2017-28 February 2019). Interrupted time series analysis were performed with Stata/SE V.15. Associated changes in payment were calculated based on published Medicare payment amounts for ESR, and changes in institutional cost were estimated based on direct cost estimates from the cost accounting system at our institution.

We performed structured chart audit of a sample of emergency department encounters to evaluate the appropriateness of ESR testing in the preintervention and postintervention period. $\chi^{2}$ analysis was used to compare the percentage of appropriate ESR tests performed in the preintervention with the postintervention period.

\section{DESIGN}

Our project team was composed of resident and attending physicians in Department of Pathology and Laboratory Medicine and in the Department of Medicine, a medical student, an EMR analyst and a quality improvement specialist. Patients or the public were not involved in the design, conduct, reporting or dissemination plans of our research. We used preliminary data regarding ESR and CRP test utilisation from inpatient and outpatient settings to identify individual providers and groups with high ESR utilisation. Physician leaders in high utilisation groups were invited to complete an email survey regarding current practices and knowledge regarding ESR and CRP ordering. Based on the survey results and literature review, we developed a three-component intervention: (1) an educational campaign, (2) development of a new order panel for ESR and CRP with point-of-order CDS in the EMR and (3) regular audit and feedback to groups with high combined ESR/CRP utilisation. After introducing the CDS in the EMR, we sought feedback from high utilisation groups and modified the order panel and CDS based on this feedback.

We anticipated several challenges in implementing the project. Although many physician leaders were amenable to learning about the project and providing feedback, successful implementation would require buy-in and changes in test ordering practices of front-line clinicians across the organisation. We recognised the need for educational approaches and communication efforts focused on specific clinician groups with high test ordering rates and also on the broader community of clinicians. Clinicians frequently expressed concerns around cumbersome clinical alerts and inefficient EMR functionality, leading the project team to prioritise non-intrusive electronic CDS options. We expected potential delays in modifying orders and developing CDS within the electronic health record due to limited resources for building EMR orders and competing institutional priorities.

\section{STRATEGY}

Prior to developing our interventions, we conducted an email survey of selected physician leaders in groups with high ESR utilisation rates to investigate reasons for the current practice. Five open-ended questions explored: (A) perceived drivers of combined ESR/CRP testing, (B) potential negative impact of reducing ESR testing, (C) clinical situations in which ESR would be preferable to CRP, (D) potential barriers to change and (E) anticipated impact of EMR CDS on the clinical practice of their group.

We performed an informal thematic analysis of survey results, which identified several recurring themes. Surveyed physicians across different specialties indicated low awareness that CRP was a more sensitive and specific test for acute phase inflammation and that the American Society for Clinical Pathology recommended against ordering an ESR in this clinical scenario. Clinical habit and ease of test ordering were identified as key drivers of ESR overuse. In addition, several surveyed physicians identified specific disease states or clinical scenarios where they felt ESR provided additive or more accurate 
clinical information than CRP, and thus, completely eliminating ESR use would not be a clinically prudent or feasible goal. One surveyed physician indicated that she preferred ESR because she (mistakenly) believed it was a less expensive test than CRP. As anticipated, there was a general concern that EMR changes would make it more difficult to order appropriate tests.

Our first Plan-Do-Study-Act (PDSA) cycle, the educational campaign, included face-to-face educational sessions with high-utiliser groups and other key clinical stakeholders. During the months of November and December 2017, we conducted educational sessions with key ordering groups including internal medicine hospitalists, internal medicine residents, family medicine residents and clinicians in rheumatology, infectious disease and emergency medicine. The evidence regarding ESR and CRP testing and practice recommendations were reviewed. Across groups, clinicians frequently reported that they were unaware of the test characteristics of ESR and CRP and of the Choosing Wisely recommendation. In response, we developed a brochure highlighting the Choosing Wisely recommendation and supporting evidence and distributed the brochure electronically to resident physicians and medical staff.

Our second PDSA cycle focused on changes to the EMR (Epic Systems Corporation). During the preintervention period, CRP and ESR were individual, separate orders in our EMR, and no guidance regarding appropriate order selection was provided within the orders. We sought to provide concise, non-intrusive CDS while making it easier to order the tests appropriately. We developed a new order panel that included both ESR and CRP. In the order panel, CRP was preselected, and language was added to recommend the use of CRP to detect acute inflammation (figure 1). Additionally, a link to the related Choosing Wisely recommendation (figure 1) was provided. Our initial electronic CDS intervention was implemented in December 2017. The individual orders for ESR and CRP were removed from preference lists and replaced with the order panel.

Based on feedback about design and use of the order panel, we updated the electronic CDS intervention in January 2018. We discovered that the order panel could not be used from telephone encounters, leading to frustration for one group of physicians who used this functionality routinely and necessitating the reactivation of individual ESR and CRP orders. At the time of reactivation, we added decision support language in each individual order. We also clarified the naming convention of the high-sensitivity CRP to reduce confusion regarding its use.

For our third PDSA cycle, we conducted audit and feedback at the divisional or group level about test frequency. We developed a report showing a run chart of the monthly number of ESR tests, CRP tests and combined ESR and CRP tests performed. Starting in April 2018, this report was sent via email to division chiefs in areas of high ESR utilisation to promote adoption and sustainability. The electronic communication included a reminder of the rationale for the project and was sent quarterly.

Following these interventions, we performed a retrospective, structured chart review to analyse ESR test appropriateness. Chart review was performed on encounters for patients presenting to the emergency department. The emergency department was selected as it represented a setting where clinical assessment for acute inflammation in patients with undiagnosed conditions would be common and where baseline utilisation of both ESR and CRP testing was observed to be high. The emergency department accounted for $15.2 \%$ of the total institutional ESR testing in the baseline period, second only to rheumatology, where ESR use was suspected to reflect testing in patients with previously diagnosed inflammatory diseases rather than patients with undiagnosed conditions. Appropriateness of ordering was assessed based on clinical documentation and the patient's history of pre-existing inflammatory conditions. We identified all patient encounters in the emergency department during which an ESR test was performed. From this list, 80 patient encounters during the preintervention period and 80 patient encounters during the postintervention period were randomly selected for structured chart review. This sample size was based on a priori calculations to detect a $25 \%$ increase in appropriateness of ESR test ordering with a power of $80 \%$ at $\alpha=0.05$. Charts were reviewed to assess for pre-existing inflammatory conditions, including rheumatological disease, active malignancy or active infectious process, as listed on the patient's problem list or within the emergency department provider and/or consulting specialist's documentation. If an inflammatory condition was listed in one or more of these areas and it could be reasonably associated with the patient's chief complaint,

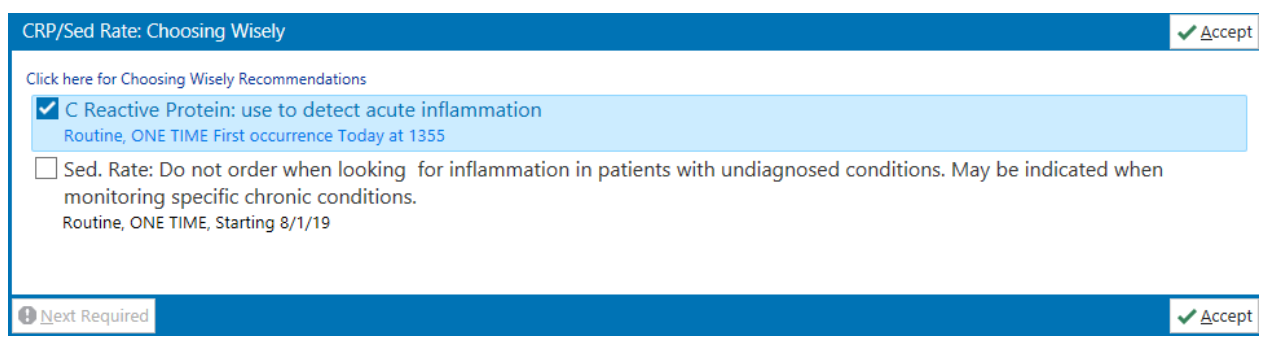

Figure 1 Electronic clinical decision support intervention as displayed in the electronic medical record. Image copyright EPIC systems Corporation. Used with permission. 
Table 1 Comparison of mean number of test per month in preintervention and postintervention periods

\begin{tabular}{|c|c|c|}
\hline \multirow[b]{2}{*}{ Test } & \multicolumn{2}{|c|}{ Mean $(95 \% \mathrm{Cl})$ tests per month } \\
\hline & Preintervention* & Postintervention† \\
\hline ESR & 667636 to 697$)$ & 447 (418 to 476$)$ \\
\hline Concurrent ESR and CRP & 504 (483 to 525$)$ & 378 (358 to 398$)$ \\
\hline CBC $\ddagger$ & 13726 (13 359 to 14093$)$ & $\begin{array}{l}14441 \text { (14 } 098 \text { to } 14 \\
785)\end{array}$ \\
\hline
\end{tabular}

*Preintervention period was from 1 September 2016 to 31 October 2017.

†Postintervention period was from 1 November 2017 to 28 February 2019.

$\ddagger$ CBC served as control.

$\mathrm{CBC}$, complete blood count; CRP, C reactive protein; ESR, erythrocyte sedimentation rate.

the ordering of ESR was deemed as appropriate. If it appeared that the ESR was ordered to evaluate for acute phase inflammation without a diagnosed inflammatory condition, the ordering of the ESR was categorised as inappropriate.

\section{RESULTS}

Table 1 presents the mean number of tests per month in the preintervention and postintervention period. Data from the 14-month preintervention period demonstrated a mean rate of $667 \mathrm{ESR}$ tests per month and 684 CRP tests per month. Of these tests, an average of 504 were ordered as combined ESR and CRP per month. In the postintervention period, there was a $33 \%$ decrease in the mean number of ESR tests per month (-219 tests per month, $\mathrm{p}<0.001)$. Similarly, there was a $25 \%$ decrease in the mean number of concurrently ordered ESR and CRP tests per month $(-126$ tests per month, $\mathrm{p}<0.001)$. No significant change in the mean number of CRP tests per month was observed $(+26$ tests per month, $p=0.23)$. Between the preintervention and postintervention periods, the mean number of CBC tests per month increased $(+715$ tests per month). Figure 2 displays a run chart of the monthly test volumes.

Interrupted time-series analysis demonstrated a significant decrease in the number of ESR orders in the first month following the intervention (coefficient -189.8 , $\mathrm{p}<0.001)$ and a significant change in the slope of the monthly number of ESR orders (coefficient -13.8, $\mathrm{p}=0.002$ ). In contrast, there was no significant change in the number of CRP orders in the first month following the intervention (coefficient $-37.6, p=0.358$ ) or the slope of the monthly number of CRP orders (coefficient -7.6 , $\mathrm{p}=0.11$ ).

The decrease in ESR tests resulted in an estimated payment reduction of $\$ 8769$ annually (based on Medicare payment amount per test) and a direct cost avoidance of \$23701 annually (based on institutional cost accounting estimates) for our institution.

In assessing appropriateness of ESR ordering preintervention and postintervention, a total of 160 charts with ESR results were randomly selected and audited (80 charts in the 6 months preintervention and 80 charts 6 months postintervention). $\chi^{2}$ testing demonstrated no significant improvement in appropriateness of ESR test ordering following the intervention (table 2). Providers were not more likely to document the reason for ordering an ESR test nor to document the results of ESR testing after intervention. Even among the 63 encounters in which ESR testing was deemed to be appropriate from a clinical standpoint based on the presence of a diagnosed inflammatory condition, 60 (95.2\%) had concurrent CRP test. Only 1 of 29 clinically appropriate ESR tests in the preintervention period and 2 of 34 clinically appropriate ESR tests in the postintervention period were ordered individually, that is, without a concurrent CRP test. The proportion of ESR tests that were abnormal (ie, elevated) before the intervention was significantly more than those that were abnormal after intervention $(p=0.02)$. In the preintervention and postintervention periods, those ESR tests that were ordered appropriately were more

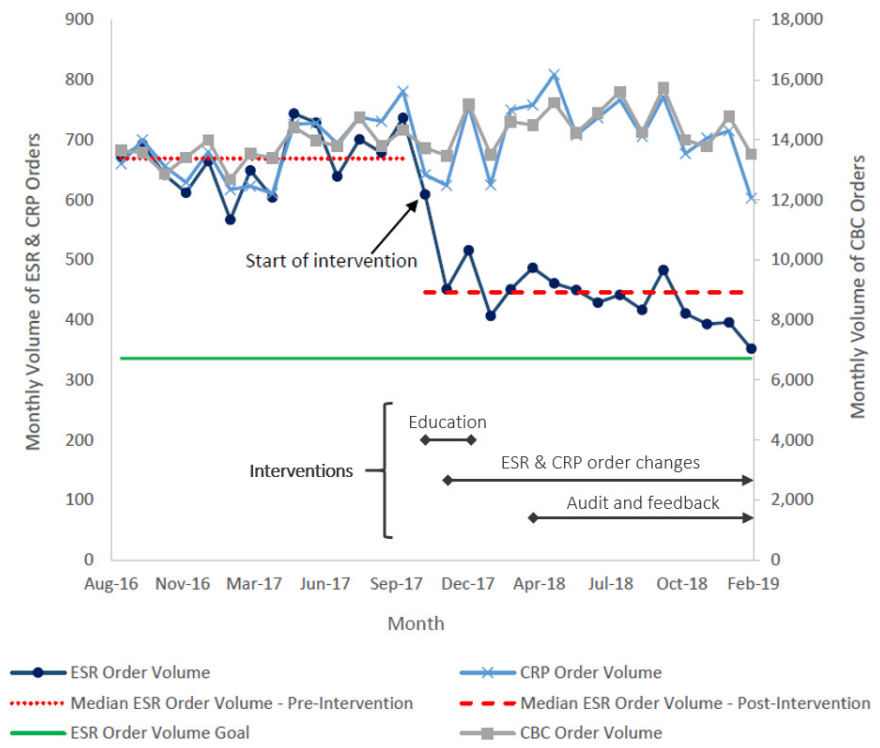

Figure 2 Run chart demonstrating numbers of ESR, $\mathrm{CRP}$ and $\mathrm{CBC}$ tests per month in the preintervention and postintervention periods. CBC, complete blood count; CRP, $\mathrm{C}$ reactive protein; ESR, erythrocyte sedimentation rate. 
Table 2 Results of structured chart audit comparing ordering and documentation practices between preintervention and postintervention periods

\begin{tabular}{|c|c|c|c|}
\hline & Preintervention, number & Postintervention, number & $P$ value \\
\hline \multicolumn{4}{|l|}{ ESR order appropriateness } \\
\hline ESR order appropriate & 29 & 34 & \multirow[t]{2}{*}{0.24} \\
\hline ESR order not appropriate & 51 & 46 & \\
\hline \multicolumn{4}{|l|}{ Documentation of reason for ordering ESR } \\
\hline Reason for ordering documented & 2 & 3 & \multirow[t]{2}{*}{0.47} \\
\hline No reason for ordering documented & 78 & 77 & \\
\hline \multicolumn{4}{|l|}{ Documentation of result of ESR test } \\
\hline ESR result documented & 42 & 34 & \multirow[t]{2}{*}{0.07} \\
\hline ESR result not documented & 38 & 46 & \\
\hline \multicolumn{4}{|l|}{ ESR test results } \\
\hline Normal ESR result & 43 & 53 & \multirow[t]{2}{*}{0.02} \\
\hline Abnormal ESR result & 37 & 27 & \\
\hline
\end{tabular}

ESR, erythrocyte sedimentation rate

likely to be abnormal than those ordered inappropriately $(\mathrm{p}<0.001)$.

\section{LESSONS AND LIMITATIONS}

We observed a significant reduction in ESR testing and combined ESR and CRP testing following an intervention incorporating education, CDS in the EMR and regular feedback about test utilisation. The strengths of the initiative included stakeholder engagement and systems changes combining CDS with efforts to improve ease of ordering CRP and ESR. The interrupted time series analysis suggests that the reductions in the ESR tests were due to the interventions and not a result of secular trends in laboratory testing. This conclusion is supported by an increase in the number of CBCs-the control test-between the preintervention and postintervention periods. The change in ESR test volumes was sustained across a prolonged postintervention period of 14 months.

Although the increase in the number of CRP tests observed did not reach statistical significance, it is possible that CRP was selected instead of ESR in some instances and may contribute to a sustained increase in utilisation of CRP over time. However, it is also possible that the non-significant increase in CRP tests reflected secular trends in patient volumes, inflammatory conditions or test ordering overall.

The calculated annual cost savings of $\$ 23701$ is based on direct cost estimates for ESR tests only. As this calculation does not include indirect costs, it likely underestimates the total cost savings. The calculated annual payment reduction of $\$ 8769$ is based on Medicare payments for ESR tests. Variability in payment rates among commercial payers and prospective payment models for hospitalised patients make the true payment impact difficult to ascertain accurately.

We did not achieve our aim of reducing combined CRP and ESR tests by $50 \%$ by 1 year. Despite a significant reduction in the number of ESR tests, structured chart review indicated that residual ESR tests during the postintervention period were still ordered inappropriately in a majority of cases. Furthermore, most ESR tests deemed to be clinically appropriate based on the presence of a diagnosed inflammatory condition were ordered concurrently with CRP tests, indicating the persistence of low value test ordering after the intervention. Since the CDS appears for all ESR tests ordered, we hypothesise that the persistently inappropriate ordering may represent some combination of factors, including ingrained practice habits, scepticism of the practice recommendations, belief that the ESR test may be useful once a diagnosis is established or failure to notice/read the embedded CDS language due to time constraints or attentional distractions. The EMR changes were intentionally designed to allow rapid ordering of inflammatory markers (CRP and ESR) while providing non-intrusive, passive guidance regarding recommended ordering practices. More active CDS, such as a required prompt to select an indication for ESR ordering, may be needed to achieve further reductions in inappropriate ESR test orders. Eliminating the ability to order ESR and CRP concurrently may help to address the persistence of this low value practice and compel clinicians to consider the preferred test in a specific clinical scenario. Restricting the privilege to order ESR to specialty areas such as rheumatology, where clinical indications for ESR testing are more likely, may enhance the effectiveness of the interventions.

As this initiative was conducted at a single academic medical centre with an integrated outpatient and inpatient EMR, the generalisability may be limited. While the decrease in ESR testing was sustained across a 14-month postintervention period and the CDS remains embedded in the EMR, it is uncertain if the decreased test utilisation will persist if education and regular feedback are discontinued. Lastly, the clinical impact of the reduction 
in ESR tests were not assessed. Although ESR is of low value in assessing acute inflammation in undiagnosed conditions, it may have clinical utility in the management of some specific clinical conditions, such as osteomyelitis, multiple myeloma and macroglobulinaemia. ${ }^{6-8}$ While there is a small potential risk that this initiative will reduce appropriate ordering of ESR tests, there is likely a greater clinical risk to patients from inappropriate ESR testing, such as false positive tests leading to unnecessary downstream testing or treatment and false negative tests leading to missed diagnostic or therapeutic opportunities. Reductions in unnecessary downstream testing and treatment may substantially decrease patient harm and institution costs. However, there is a paucity of literature on the frequency, costs and clinical impact of downstream testing and treatment following inappropriate ESR testing. Therefore, we did not include estimates of these potential downstream costs and savings in our financial modelling.

\section{CONCLUSION}

A simple, reproducible, multifaceted initiative was associated with sustained reductions in ESR tests and modest decreases in institutional costs. However, inappropriate ESR test ordering persisted following the intervention, suggesting opportunities for continued improvement and the possible need for more active CDS.

Acknowledgements We gratefully recognise the assistance of Alicia Cardoza with developing order panels and clinical decision support, Rhonda Kost with obtaining data, and Jeffrey Priest, PhD, in Biomedical Statistics, and Bradley Tompkins, MS, $\mathrm{MPH}$, in the Department of Medicine Quality Program with performing statistical analyses.

Contributors KJB participated in chart review/data collection and analyses and led the manuscript development. APV participated in the conception, design, implementation, analyses and manuscript editing. JR participated in the design, implementation, analyses and manuscript editing. CW, EHS and JS-D participated the design, implementation, analyses, and manuscript editing. ABR supervised the conception, design, implementation, analyses and manuscript development and submission. AR accepts full responsibility for the overall content and conduct of the study.

Funding The authors have not declared a specific grant for this research from any funding agency in the public, commercial or not-for-profit sectors.

Competing interests None declared.

Patient consent for publication Not required.

Ethics approval The Research Protections Office at the University of Vermont determined that the project did not meet the definition of research activity adopted by the University of Vermont Institutional Review Board.

Provenance and peer review Not commissioned; externally peer reviewed.

Data availability statement Data are available upon request.

Open access This is an open access article distributed in accordance with the Creative Commons Attribution Non Commercial (CC BY-NC 4.0) license, which permits others to distribute, remix, adapt, build upon this work non-commercially, and license their derivative works on different terms, provided the original work is properly cited, appropriate credit is given, any changes made indicated, and the use is non-commercial. See: http://creativecommons.org/licenses/by-nc/4.0/.

ORCID iD

Allen B Repp http://orcid.org/0000-0001-7513-532X

\section{REFERENCES}

1 Colombet I, Pouchot J, Kronz V, et al. Agreement between erythrocyte sedimentation rate and $\mathrm{C}$-reactive protein in hospital practice. Am J Med 2010;123:863.e7-13.

2 Choosing Wisely Campaign. American Society of clinical pathology recommendations. Available: https://www.choosingwisely.org/ societies/american-society-for-clinical-pathology/ [Accessed 1 Aug 2019].

3 Harrison M. Erythrocyte sedimentation rate and C-reactive protein. Aust Prescr 2015;38:93-4.

4 Kainth MK, Gigliotti F. Simultaneous testing of erythrocyte sedimentation rate and C-reactive protein: increased expenditure without demonstrable benefit. J Pediatr 2014;165:625-7.

5 Gottheil S, Khemani E, Copley K, et al. Reducing inappropriate ESR testing with computerized clinical decision support. BMJ Qual Improv Rep 2016;5:1.

6 Lin Z, Vasudevan A, Tambyah PA. Use of erythrocyte sedimentation rate and C-reactive protein to predict osteomyelitis recurrence. $J$ Orthop Surg 2016;24:77-83.

7 Koshiaris C, Van den Bruel A, Oke JL, et al. Early detection of multiple myeloma in primary care using blood tests: a case-control study in primary care. Br J Gen Pract 2018;68:e586-93.

8 Morra E, Cesana C, Klersy C, et al. Clinical characteristics and factors predicting evolution of asymptomatic IgM monoclonal gammopathies and IgM-related disorders. Leukemia 2004;18:1512-7. 